\title{
Recours À L'avortement Provoqué Chez Les Femmes En Union Des Quartiers Précaires De Yopougon À Abidjan
}

\author{
Doumbia Mohamed, enseignant-chercheur \\ Université de Cocody-Abidjan,UFR Sciences de l'Homme et de la Société, \\ Institut d'Ethnosociologie, Côte d'Ivoire \\ Centre Suisse de Recherches Scientifiques en Côte d'Ivoire, Abidjan
}

doi: 10.19044/esj.2016.v12n33p319 URL:http://dx.doi.org/10.19044/esj.2016.v12n33p319

\begin{abstract}
In Côte d'Ivoire, $15 \%$ of maternal deaths are related to illegal abortions. Despite the known consequences, abortion practice is increasing more and more in a restrictive legislative context. Few formal studies and national statistics on the phenomenon exist, much less at the level of precarious slums of Abidjan.

This article is a contribution to the understanding of abortion practice in the unprivileged urban context of Yopougon in Abidjan. It aims to analyze the logics and conditions of abortion in the precarious slums of Yopougon.

A survey of a sample of 309 women aged 15 to 49 years, living in union in six precarious slums and, an investigation in three public health facilities and five private clinics and among traditional healers were conducted.

Results indicate an overall prevalence of $11 \%$.

Women who aborted are generally young (85.3\%), with no education (35.30\%) or primary level of education (32.35\%). Proportion of women who aborted increase with parity up to three children and decreases from the fourth child. The lack of financial resources (79.41\%) and the fear and stigma (47.05\%) are the main reasons of abortion. The majority of abortions took place in private clinics (58.82\%).
\end{abstract}

Keywords: Abortion, Precarious slums, Abidjan, Côte d'Ivoire

Résumé

En côte d’Ivoire, 15\% des décès maternels sont liés aux avortements clandestins. En dépit des conséquences dramatiques, la pratique de l'avortement prend de plus en plus d'ampleur en Côte d'Ivoire et ce, dans un contexte législatif restrictif. Peu de statistiques officielles et d'études 
nationales existent sur le phénomène et encore moins au niveau des quartiers précaires d'Abidjan.

Cet article est une contribution à la compréhension de la pratique de l'avortement dans le contexte urbain défavorisé de Yopougon à Abidjan. Il a pour objectif d'analyser les logiques qui sous-tendent le recours à l'avortement ainsi que les conditions d'avortement dans les quartiers précaires de Yopougon.

Une enquête transversale par questionnaire auprès d'un échantillon de 309 femmes de 15 à 49 ans vivant en union dans six quartiers précaires, une enquête transversale par questionnaire dans trois structures sanitaires publiques et cinq les cliniques privées et des entretiens semi-directifs avec des tradi-praticiens ont été réalisées.

Les résultats indiquent une prévalence générale de 11\%. Les femmes qui ont recours à l'avortement sont généralement jeunes (85,3\%), sans instruction (35,30\%) ou de niveau primaire (32,35\%). La proportion des avortées augmentent avec la parité jusqu'à trois enfants et diminue à partir du quatrième enfant. Le manque de ressources financières $(79,41 \%)$ et la peur et la stigmatisation $(47,05 \%)$ sont les principales raisons du recours à l'avortement.

La majorité des avortements a eu lieu dans les cliniques privées (58,82\%)

Mots clés: Avortement provoqué, Quartiers précaires, Abidjan, Côte d'Ivoire

\section{Introduction}

En Afrique, la pratique de l'avortement est une importante cause de morbidité et de mortalité. Selon l'OMS (2007), un décès maternel sur sept en Afrique est le résultat d'un avortement non médicalisé. Dans bien des cas, les conséquences de la pratique sont peu mesurées par les femmes.

Les niveaux élevés de mortalité liée à l'avortement en Afrique sont généralement le fait de l'accès limité aux services de santé reproductive, au recours à des avortements à risque, mais aussi et surtout au contexte social et légal contraignant des avortements (Hord et al., 2006; Silberschmidt et Rasch, 2001).

Les avortements en Afrique se font en effet dans un contexte législatif et réglementaire souvent restrictif. 99\% des avortements pratiqués se feraient dans l'illégalité (Guillaume, 2005) et 92\% des femmes en âge de procréer en Afrique vivent dans des pays où la législation en matière d'avortement est restrictive (Institut Guttmacher, 2009)

Les avortements en Afrique sont également pratiqués dans des conditions d'hygiène et de sécurité insuffisantes, ce qui expose les femmes à des complications post-avortements sévères. Par ailleurs, la crainte d'une 
stigmatisation sociale, la peur des sanctions légales ou religieuses suite à un avortement, conduisent les femmes en général et les adolescentes et jeunes femmes en particulier, à prendre des risques sanitaires en avortant clandestinement (Plummer et al., 2008 cités par N’Bouké, 2011).

En côte d'Ivoire, l'on a enregistré en 2005, 543 décès maternels pour 100.000 naissances vivantes dont $13 \%$ liés aux avortements clandestins (EIS, 2005). Ce rapport de mortalité maternelle est passé à 614 décès pour 100000 naissances en 2012 dont 15\% liés aux complications des avortements (EDSMICS, 2012).

La pratique de l'avortement est illégale en Côte d'ivoire, mais autorisée uniquement lorsqu'il s’agit de sauver la vie de la femme. Même à ce niveau, le recours à l'avortement est conditionné par le consentement du médecin traitant et de deux autres médecins selon le code pénal ivoirien ${ }^{14}$

Les études menées en Côte d'Ivoire sur la pratique de l'avortement montrent que les femmes en milieu urbain sont plus susceptibles de recourir à l'avortement que celles du milieu rural (Desgrées du Loû et al, 1999, Coulibaly 2009). Au niveau d'Abidjan, l'étude menée par Guillaume et Desgrées du Loû (2002) auprès de 2400 femmes d'Abidjan sur leurs antécédents contraceptifs et abortifs a montré que la proportion des femmes qui ont recours à l'avortement est plus importante chez celles qui ont un niveau d'étude secondaire (51\%) comparée aux non scolarisées (18\%) et à celles qui ont un niveau d'étude primaire (36\%). Parmi les femmes interrogées, 30\% ont déclaré avoir eu recours à l'avortement

L'intensité du recours à l'avortement varie également selon les caractéristiques socio-économiques des femmes : les femmes non mariées, les femmes d'un niveau d'instruction secondaire et supérieur et les jeunes femmes sont celles qui ont le plus recours à l'avortement (Guillaume 2000). Quant à leurs motivations, elles sont liées à la crainte des parents et à la stigmatisation de la société, au poids de la responsabilité du fait de la jeunesse et au manque de moyens financiers (Coulibaly, op.cit).

\footnotetext{
${ }^{14}$ Le code pénal ivoirien en son Titre II, Chapitre 3, à la Section 3 en son article 367 stipule que «Il n'y a pas d'infraction lorsque l'interruption de la grossesse est nécessitée par la sauvegarde de la vie de la mère gravement menacée. Dans ce cas, le médecin traitant ou le chirurgien doit obligatoirement prendre l'avis de deux médecins consultants, qui, après examen et discussion, attesteront que la vie de la mère ne peut être sauvegardée qu'au moyen d'une telle intervention chirurgicale ou thérapeutique.

- Si le nombre de médecin résidant au lieu de l'intervention est de deux, le médecin traitant n'est tenu de prendre que l'avis de son confrère ;

- Si le médecin traitant est seul résidant au lieu de l'intervention il atteste sur son honneur que la vie de la mère ne pouvait être sauvegardée que par l'intervention chirurgicale ou thérapeutique utilisée.

- Dans tous les cas, un des exemplaires de la consultation est remis à la mère, l'autre est conservé par le ou les médecins traitants.
} 
Bien que la pratique de l'avortement prenne de l'ampleur et ce, en dépit de la connaissance des conséquences par les femmes, l'absence de statistiques officielles et d'études d'envergure nationale, ne permet pas de mesurer la prévalence du phénomène au niveau national (Bi Vroh et al, 2012) et encore moins dans les quartiers précaires notamment d'Abidjan.

Cette étude est une contribution à la compréhension du phénomène dans le contexte urbain défavorisé de la commune de Yopougon à Abidjan.

L'objectif de cet article est d'analyser les logiques qui sous-tendent le recours à l'avortement ainsi que les conditions d'avortement dans les quartiers précaires de Yopougon.

De façon spécifique, il s’agit

- $\quad$ (i) d'identifier les motivations et les modalités de prises de décisions

- $\quad$ (ii) décrire les conditions de réalisation des avortements

\section{Méthodologie \\ Zone d'étude}

L’étude a été menée à Yopougon, la commune vaste du district d'Abidjan et de la Côte d'Ivoire. Avec une superficie de $85 \mathrm{Km}^{2}$, sa population qui était de 688.235 habitants au recensement général de la population de 1998 (INS, 1998), est estimée aujourd'hui à plus d'un million d'habitants. C'est également à Yopougon que se trouve la majorité des quartiers précaires (18 au total). La collecte des données a été faite dans six de ces quartiers précaires de la commune à savoir Doukouré, Gbinta, Mamie Faitai, Niangon continu, Yamoussoukro et Yaoséhi. Ces 6 quartiers ont la particularité d'être traversés par un grand canyon qui part de la zone industrielle de Yopougon à la lagune Ebrié.

\section{Sources de données et approche méthodologique}

Les données de cette étude proviennent

- $\quad$ d'une enquête ménage effectuée dans les quartiers précaires ;

- d'une enquête dans (i) trois structures sanitaires publiques de la commune de Yopougon à savoir le Centre Hospitalier Universitaire (CHU) de Yopougon, l'Association pour la Gestion de la Formation Sanitaire à base communautaire de Yopougon Niangon (AGEFOSYN) et la Formation Sanitaire Urbaine à base Communautaire (FSU-Com) de Yopougon devenue Hôpital Général de Yopougon Attié, et (ii) cinq cliniques privées et,

d'une enquête auprès de tradi praticiens.

L'approche de la recherche a été à la fois qualitative et quantitative. $\mathrm{Au}$ niveau qualitatif des entretiens semi directifs, des Focus Group Discussions et des observations directes ont été menés. Les entretiens ont été menés avec les responsables et le personnel des structures sanitaires publiques et privées et les tradi-praticiens. Les Focus Group Discussions ont 
été menées avec les associations de femmes et de jeunes. Quant aux observations, elles ont été faites au sein des structures sanitaires publiques et privées.

$\mathrm{Au}$ niveau quantitatif, des entretiens par questionnaires ont été réalisés au sein des ménages.

\section{Echantillonnage et traitement des données}

L’échantillonnage s’est appuyé sur la base de données du Recensement Général de la Population et de l'Habitat (RGPH) de 1998 de l’Institut National de la Statistique (INS).

Des six quartiers, l’on dénombrait 12184 ménages, pour 20919 hommes et 19730 femmes dont 12633 femmes en âge de procréer soit 31\% de la population totale.

La détermination de notre échantillon s'est faite sur la base d'une précision de 5\% et d'un seuil de confiance de 95\%.

Ainsi, la taille minimale de l'échantillon a été déterminée par l'application de la formule suivante:

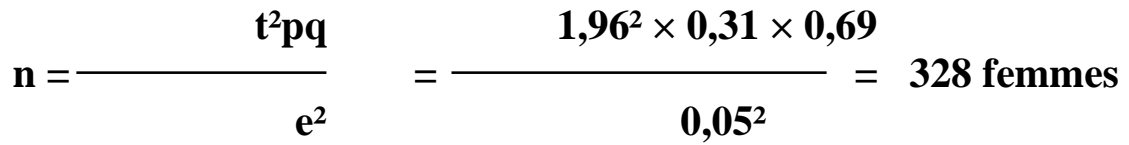

Avec les biais de remplissage et les refus, en définitive, ce sont 309 femmes et 305 hommes qui ont été interrogés.

Les femmes et les hommes ont été interrogés séparément et le critère d’inclusion dans l'échantillon étaient le fait de vivre maritalement (mariée légalement, coutumièrement, religieusement ou en concubinage) dans un ménage avec un homme. Ainsi, les ménages dans lesquels se trouvaient des femmes en âge de procréer avec ou sans enfant et vivant maritalement ont été pris en compte.

\section{Traitement et analyse de données}

Les données quantitatives ont été saisies, traitées et analysées à l’aide du logiciel SPSS.12.

Le test de Chi-2 a été utilisé et le seuil de significativité retenu était une valeur de $\mathrm{p}<0,05$.

Quant aux données qualitatives, elles ont été saisies sous Word et transportées vers le logiciel Maxqda pour traitement. Elles ont ensuite fait l'objet d'une analyse de contenu.

\section{Limite de l'étude}

Bien que l'étude ait porté sur 309 femmes, les analyses relatives à l'avortement ont porté essentiellement sur les femmes qui ont contracté au moins une grossesse non désirée au cours de leurs vie féconde et 
particulièrement celles qui ont subi un avortement. Cette analyse par « déduction » a réduit considérablement l’échantillon sur lequel l’analyse approfondie a porté en définitive. En effet, la taille de l'échantillon parait très petite $(n=34)$ par rapport au phénomène pertinent de l’avortement rapporté à l'échelle d’une commune. Bien qu'elle rende compte de la prévalence de l'avortement provoqué dans ces quartiers, une taille plus grande aurait certainement permis de saisir et d'expliquer davantage le phénomène et son impact.

Cependant, la complexité du phénomène et surtout son caractère clandestin et intime peuvent justifier la faiblesse de l’échantillon.

\section{Résultats}

\section{Prévalence de l'avortement et caractéristiques des enquêtées}

L'avortement est une pratique qui est désapprouvée par l'ensemble des femmes enquêtées $(98,7 \%, n=309)$. Mais cette désapprobation quasi unanime tranche avec la pratique de l'avortement elle même. En effet, sur les 309 femmes interrogées, 106 femmes (34,4\%) ont contracté au moins une grossesse non désirée et parmi ces dernières (34 femmes soit 32\%) ont eu recours à l'avortement provoqué soit une prévalence générale de $11 \%$.

Parmi celles qui n’ont pas avorté $(n=72), 81,94 \%$ ont systématiquement refusé d'avorter, $8,33 \%$ ont essayé sans succès d'avorter et $9,73 \%$ ont tenté d'avorter pour ensuite renoncer, soit une femme sur deux qui a avorté ou tenter d'avorter (Tableau 1).

Tableau 1 : caractéristiques des femmes ayant contracté au moins une grossesse non désirée et des avortées.

\begin{tabular}{|c|c|c|c|c|c|}
\hline $\begin{array}{c}\text { Caractéristiques } \\
\text { socio- } \\
\text { démographiques }\end{array}$ & \multicolumn{5}{|c|}{ Attitude vis-à-vis de la grossesse non désirée } \\
\cline { 2 - 6 } & J'ai avorté & $\begin{array}{c}\text { J'ai gardé } \\
\text { la } \\
\text { grossesse }\end{array}$ & $\begin{array}{c}\text { J'ai essayé } \\
\text { d'avorter sans } \\
\text { succès }\end{array}$ & $\begin{array}{c}\text { J'ai essayé } \\
\text { d'avorter pour } \\
\text { après renoncer }\end{array}$ & TOTAL \\
\hline & $\begin{array}{c}\text { Effectif } \\
(\%)\end{array}$ & $\begin{array}{c}\text { Effectif } \\
(\%)\end{array}$ & Effectif $(\%)$ & Effectif (\%) & $\begin{array}{c}\text { Effectif } \\
(\%)\end{array}$ \\
\hline Age & & & & & \\
$15-19$ & $2(5,88)$ & $1(1,70)$ & $0(0,00)$ & $0(0,00)$ & $3(2,83)$ \\
$20-24$ & $4(11,77)$ & $16(27,12)$ & $2(33,33)$ & $0(0,00)$ & $22(20,75)$ \\
$25-29$ & $18(52,94)$ & $18(30,51)$ & $4(66,64)$ & $4(57,14)$ & $44(41,51)$ \\
$30-34$ & $7(20,59)$ & $13(22,03)$ & $0(0,00)$ & $2(28,57)$ & $22(20,75)$ \\
$35-39$ & $3(8,82)$ & $6(10,17)$ & $0(0,00)$ & $0(0,00)$ & $9(8,49)$ \\
$40-44$ & $0(0,00)$ & $5(8,47)$ & $0(0,00)$ & $0(0,00)$ & $5(4,72)$ \\
$45-49$ & $0(0,00)$ & $0(0,00)$ & $0(0,00)$ & $1(14,29)$ & $1(0,95)$ \\
\hline Niveau d'instruction & & & & & \\
Aucun & $12(35,30)$ & $18(30,50)$ & $3(50,00)$ & $2(28,57)$ & $35(33,02)$ \\
Préscolaire & $0(0,0)$ & $1(1,70)$ & $0(0,0)$ & $1(14,29)$ & $2(1,89)$ \\
Primaire & $11(32,35)$ & $29(49,15)$ & $1(16,67)$ & $2(28,57)$ & $43(40,57)$
\end{tabular}




\begin{tabular}{|c|c|c|c|c|c|} 
Secondaire & $11(32,35)$ & $10(16,95)$ & $2(33,33)$ & $2(28,57)$ & $25(23,58)$ \\
Supérieur & $0(0,0)$ & $1(1,70)$ & $0(0,00)$ & $0(0,00)$ & $1(0,94)$ \\
\hline Situation & & & & & \\
professionnelle & & & & & \\
Occupée & $21(61,77)$ & $35(59,32)$ & $1(16,67)$ & $3(42,86)$ & $60(56,60)$ \\
Ménagère & $8(23,53)$ & $21(35,59)$ & $4(66,66)$ & $3(42,86)$ & $36(33,96)$ \\
Ménagère/Occupée & $3(8,82)$ & $3(5,09)$ & $0(0,00)$ & $1(14,28)$ & $7(6,603)$ \\
Elève/étudiant & $1(2,94)$ & $0(0,0)$ & $1(16,67)$ & $0(0,00)$ & $2(1,89)$ \\
Inactive & $1(2,94)$ & $0(0,0)$ & $0(0,00)$ & $0(0,00)$ & $1(0,95)$ \\
\hline Religion & & & & & \\
Chrétien & $25(73,53)$ & $36(61,02)$ & $3(50,00)$ & $7(100)$ & $71(66,98)$ \\
Musulman & $4(11,76)$ & $7(11,864)$ & $2(33,33)$ & $0(0,00)$ & $13(12,27)$ \\
Sans religion & $5(14,71)$ & $16(27,12)$ & $1(16,67)$ & $0(0,00)$ & $22(20,75)$ \\
\hline Groupe d'ethnique & & & & & \\
Akan & $11(32,35)$ & $(25,423)$ & $1(16,67)$ & $3(42,86)$ & $30(28,30)$ \\
Krou & $10(29,41)$ & $18(30,51)$ & $0(0,00)$ & $4(57,14)$ & $32(30,19)$ \\
Mandé du nord & $4(11,77)$ & $5(8,48)$ & $3(50,00)$ & $0(0,00)$ & $12(11,32)$ \\
Mandé du sud & $9(26,47)$ & $18(30,51)$ & $2(33,33)$ & $0(0,00)$ & $29(27,36)$ \\
Non ivoirienne & $0(0,0)$ & $3(5,08)$ & $0(0,00)$ & $0(0,00)$ & $3(2,83)$ \\
\hline Nombre d'enfant & & & & & \\
0 & $5(14,71)$ & $2(3,39)$ & $1(16,67)$ & $0(0,00)$ & $8(7,55)$ \\
1 & $9(26,47)$ & $18(30,51)$ & $2(33,33)$ & $1(14,29$ & $30(28,30)$ \\
2 & $12(35,30)$ & $19(32,21)$ & $3(50,00)$ & $2(28,57)$ & $36(33,96)$ \\
Plus de 5 enfants & $0(0,0)$ & $3(5,08)$ & $0(0,00)$ & $0(0,00)$ & $3(2,83)$ \\
\hline TOTAL & $34(100)$ & $59(100)$ & $6(100)$ & $7(100)$ & $106(100)$ \\
\hline
\end{tabular}

L'analyse des caractéristiques sociodémographiques des femmes qui ont eu recours à l'avortement provoqué montre que le phénomène s'observe pratiquement à tous les âges, quels que soient le niveau d'instruction, la parité ou la situation professionnelle.

Au niveau de l'âge, les femmes dont l'âge se situe entre 20 ans et 34 ans sont les plus nombreuses à avoir eu recours à l'avortement $(85,3 \%)$ avec un pic élevé au niveau des 25-29 ans (52,94\%). Ce qui signifie que les jeunes filles ont beaucoup plus recours à l'avortement que leurs aînées.

$\mathrm{Au}$ niveau de l'instruction, les femmes qui n'ont aucun niveau d'instruction (35,30\%) et celles qui ont le niveau primaire $(32,35 \%)$ sont les plus nombreuses à avoir contracté une grossesse non désirée $(75,47 \%)$, mais aussi à avoir avorté (67,65\%). Cependant, si l'on raisonne en terme de moyenne, c'est au niveau des femmes qui ont atteint le niveau secondaire que le nombre moyen d'avortées est le plus significatif. Sur les 25 femmes du niveau secondaire, 11 ont avorté et 10 ont gardé leur grossesse alors que 
sur les 35 femmes qui n’ont aucun niveau d’instruction, 12 ont avorté contre 18 qui ont gardé leur grossesse. Chez celles qui ont un niveau primaire par contre, 11 femmes sur les 43 au total ont interrompu leur grossesse contre 29 qui ont systématiquement gardé leur grossesse.

Cela pourrait s’expliquer certainement par le fait que les femmes qui ont un niveau d'instruction élevé peuvent accéder facilement aux structures sanitaires pour se faire avorter parce qu'ayant sans nul doute reçu des informations auparavant sur la planification familiale.

Cette « inégalité » entre les femmes face à l'avortement s’observe également au niveau du statut socio-professionnel. Si d'emblée, l’on peut supposer que les femmes « pauvres » ont plus recours à l'avortement parce qu'elles n’ont pas accès aux méthodes contraceptifs, donc les utilisent, les résultats de l'étude montrent au contraire que ce sont les femmes dites « occupées » c'est-à-dire celles qui mènent une activité génératrice de revenu qui ont le plus recours à l'avortement (61,77\%). Là encore, cela pourrait s'expliquer par le fait qu'elles ont les ressources financières pour se faire avorter.

Au niveau de la religion, les femmes musulmanes sont celles qui ont le moins recours à l'avortement, comparativement aux femmes des autres religions (11,76\% contre $73,53 \%$ de chrétiennes et $14,71 \%$ de sans religion). Le fait qu'il y ait une faible proportion de musulmanes qui ont recours à l'avortement pourrait, à priori, s'expliquer par la rigidité de l'islam en matière d'avortement.

Au niveau de la parité, l’on remarque que la proportion des avortées augmentent avec la parité jusqu’à trois enfants et diminue considérablement à partir du quatrième enfant.

Les tests effectués ont montré une forte présomption de liaison entre la parité des femmes et leur âge $(\chi 2=627,426 ; p=0,000)$.

\section{Intensité du recours à l'avortement}

Le recours à l'avortement chez les femmes n'est pas intense. La majorité $(67,65 \%)$ n'a eu recours à l'avortement qu'une seule fois contre 23,53\% qui ont avorté deux fois. Seulement 8,82\% des femmes ont avorté trois fois et plus (Tableau 2). 
Tableau 2 : Intensité du recours à l'avortement

\begin{tabular}{|c|c|c|c|c|}
\hline \multirow[t]{2}{*}{$\begin{array}{l}\text { Caractéristiques socio- } \\
\text { démographiques }\end{array}$} & \multicolumn{4}{|c|}{ Intensité du recours à l'avortement } \\
\hline & Une fois & Deux fois & $\begin{array}{c}\text { Trois fois et } \\
\text { plus }\end{array}$ & TOTAL \\
\hline & $\begin{array}{c}\text { Effectif } \\
(\%)\end{array}$ & $\begin{array}{c}\text { Effectif } \\
(\%)\end{array}$ & Effectif (\%) & $\begin{array}{c}\text { Effectif } \\
(\%)\end{array}$ \\
\hline Age & & & & \\
\hline $15-19$ & $2(8,70)$ & $0(0,00)$ & $0(0,00)$ & $2(5,88)$ \\
\hline $20-24$ & $4(17,39)$ & $0(0,00)$ & $0(0,00)$ & $4(11,77$ \\
\hline $25-29$ & $13(56,52)$ & $5(62,50)$ & $1(33,33)$ & $19(55,88)$ \\
\hline $30-34$ & $2(8,70)$ & $2(25,00)$ & $2(66,67)$ & $6(17,65)$ \\
\hline $35-39$ & $2(8,70)$ & $1(12,50)$ & $0(0,00)$ & $3(8,82)$ \\
\hline $40-44$ & $0(0,00)$ & $0(0,00)$ & $0(0,00)$ & $0(0,00)$ \\
\hline $45-49$ & $0(0,00)$ & $0(0,00)$ & $0(0,00)$ & $0(0,00)$ \\
\hline Niveau d'instruction & & & & \\
\hline Aucun & $8(34,78)$ & $5(62,50)$ & $1(33,33)$ & $14(41,18)$ \\
\hline Préscolaire & $0(0,00)$ & $0(0,00)$ & $0(0,00)$ & $0(0,00)$ \\
\hline Primaire & $8(34,78)$ & $2(25,00)$ & $1(33,33)$ & $11(32,35)$ \\
\hline Secondaire & $7(30,44)$ & $1(12,50)$ & $1(33,33)$ & $9(26,47)$ \\
\hline Supérieur & $0(0,00)$ & $0(0,00)$ & $0(0,00)$ & $0(0,00)$ \\
\hline Nombre d'enfant & & & & \\
\hline 0 & $6(26,08)$ & $0(0,00)$ & $0(0,00)$ & $6(17,65)$ \\
\hline 1 & $5(21,74)$ & $2(25,00)$ & $1(33,33)$ & $8(23,53)$ \\
\hline 2 & $9(39,13)$ & $2(25,00)$ & $1(33,33)$ & $12(35,29)$ \\
\hline 3 & $1(4,35)$ & $1(12,50)$ & $1(33,33)$ & $3(8,83)$ \\
\hline 4 & $1(4,35)$ & $3(37,5)$ & $0(0,00)$ & $4(11,76)$ \\
\hline 5 & $1(4,35)$ & $0(0,00)$ & $0(0,00)$ & $1(2,94)$ \\
\hline Plus de 5 enfants & & & & \\
\hline TOTAL & $23(67,65)$ & $8(23,53)$ & $3(8,82)$ & $34(100)$ \\
\hline
\end{tabular}

L'analyse des caractéristiques sociodémographiques des femmes en rapport avec l'intensité du recours à l'avortement montrent que l'intensité du recours à l'avortement semble augmenter avec l'âge. En effet, les femmes dont l'âge se situe entre 20 ans et 29 ans (73,91\%), sont celles qui ont eu moins recours à l'avortement, seulement une fois.

Par contre, l'âge de celles qui ont eu recours à l'avortement plus de trois fois se situe entre 29 ans et 34 ans. Les tests effectués ont montré des présomptions de liaison entre l'intensité du recours à l'avortement et l'âge des femmes: $(\chi 2=62,611 ; p=0,021)$

$\mathrm{Au}$ niveau des autres caractéristiques sociodémographiques, celles qui ont le plus avorté se trouvent parmi celles qui n'ont aucun niveau d'instruction, celles qui ont un niveau primaire (100\%) et celles qui ont une parité d'un à trois enfants (100\%). 
Même si l'intensité du recours à l'avortement reste faible chez les femmes, il n'en demeure pas moins que des risques de grossesses non désirées subsistent. En effet, seulement 18 des 34 femmes (52,94\%) qui ont eu recours à l'avortement utilisaient une méthode contraceptive au moment de l'enquête (Tableau 3).

Tableau 3 : Utilisation actuelle d'une méthode contraceptive par les femmes ayant eu recours à l'avortement.

\begin{tabular}{|c|c|c|c|c|}
\hline \multirow{2}{*}{$\begin{array}{c}\text { Utilisation d'une méthode } \\
\text { contraceptive au moment de } \\
\text { l'enquête }\end{array}$} & \multicolumn{2}{|c|}{ Intensité du recours à l'avortement } & \multirow{2}{*}{} \\
\cline { 2 - 4 } & Une fois & Deux fois & $\begin{array}{c}\text { Trois fois et } \\
\text { plus }\end{array}$ & Total \\
\hline OUI & 12 & 4 & 2 & 18 \\
NON & 11 & 4 & 1 & 16 \\
TOTAL & 23 & 8 & 3 & 34 \\
\hline
\end{tabular}

\section{Raisons du recours à l'avortement et prise de décision d'avorter Raison du recours à l'avortement}

L'analyse des raisons évoquées pour justifier le recours à l'avortement permet de mieux cerner le comportement des femmes (Tableau 4).

Tableau 4 : Raisons du recours à l'avortement

\begin{tabular}{|c|c|}
\hline Raison & Effectif (\%) \\
\hline La peur/la stigmatisation & $16(47,05)$ \\
Le manque de ressources financières & $27(79,41)$ \\
« Je n'étais pas prête » & $10(29,41)$ \\
La famille & $2(5,88)$ \\
" Avait un bébé sous le bras » & $6(17,64)$ \\
Les études & $3(8,82)$ \\
Les problèmes de santé & $2(5,88)$ \\
Autres & $2(5,88)$ \\
\hline
\end{tabular}

La pauvreté est la première cause d'avortement évoquée par les femmes. $79,41 \%$ d'entre elles ont évoqué le manque de ressources financières pour faire face à la survenue d'un enfant.

La seconde raison est liée à la peur et à la stigmatisation $(47,05 \%)$. L'annonce d'une grossesse non désirée associée au regard des autres contribuent à influencer le recours à $\mathrm{l}$ 'avortement selon les femmes.

A côté de ces deux principales raisons, pour les autres femmes, l'avortement se justifiait par le fait qu'au moment de la contraction de cette grossesse, elles n'étaient pas "prêtes» à assumer des responsabilités de mère (29,41\%). Ce refus de responsabilisation de ces femmes laisse penser qu'elles veulent repousser pour plus tard leur maternité. Par ailleurs, 17,84\% ont évoqué qu'elles étaient encore nourrices au moment de la contraction de la grossesse. "J'avais un bébé sous le bras » comme elles disent. Cela qui laisse entrevoir un besoin manifeste en matière de planification familiale. 


\section{Prise de décision du recours à l’avortement}

L'analyse des données permet de constater que les femmes dans leur majorité (61,76\%) avaient décidé d'elles-mêmes d'avorter lors de leur première grossesse non désirée. Pour le reste, elles l'ont fait, soit sur la contrainte du partenaire, du mari ou de l'auteur de la grossesse (23,53\%), soit sur recommandation d'un(e) ami (e) (Tableau 5).

Tableau 5 : Prise de décision

\begin{tabular}{|c|c|}
\hline Qui vous a conseillé d'avorter ? & Effectif (\%) \\
\hline Ami(e) & $5(14,71)$ \\
Personne (Moi-même) & $21(61,76)$ \\
Mari/Auteur de la grossesse & $8(23,53)$ \\
\hline
\end{tabular}

Pour celles qui ont eu recours plus d'une fois à l'avortement, la décision d'avorter pour la deuxième fois est venue d'elles-mêmes dans $37,50 \%$ des cas, d'un(e) ami(e) dans $37,50 \%$ des cas et de l'auteur ou du partenaire dans $25 \%$ des cas $(n=8)$.

Quant à celles qui ont avorté plus de deux fois, elles toutes l'ont fait sur décision personnelle.

Les tests effectués ont montré une présomption de liaison entre la prise de décision et la religion : $(\chi 2=13,106 ; p=0,041)$.

En termes de consentement mutuel dans les prises de décisions d'avorter, on note que $58,10 \%$ des femmes qui ont avorté pour la première fois, ont reçu l'accord de leur partenaire.

Par ailleurs, lors de leur première expérience d'avortement, 67,70\% des femmes ont déclaré connaître les conséquences liées à cette pratique avant de s'engager.

\section{Conditions d'avortement}

\section{Lieux d'avortement}

Trois principaux lieux ont été cités par les femmes. Il s'agit des cliniques privées, chez les tradi-praticiens et les hôpitaux publics. Les cliniques sont les lieux où la majorité des avortements (58,82\%) ont été pratiqués. Les hôpitaux publics viennent en seconde position avec 17,65\% des cas d'avortement et les locaux des tradi-praticiens (14,71\%). (Tableau 6).

On note par ailleurs que $8,82 \%$ des femmes ont avorté à domicile

\begin{tabular}{|c|c|}
\multicolumn{2}{|c|}{ Tableau 6 : Répartition des femmes selon le lieu d'avortement } \\
\hline Lieu d'avortement & Effectif $(\%)$ \\
\hline Clinique privée & $20(58,82)$ \\
Chez un tradi-praticien & $5(14,71)$ \\
Hôpital public & $06(17,65)$ \\
A la maison & $03(8,82)$ \\
\hline
\end{tabular}


Le fait que la majorité des avortements ait été pratiqué dans des cliniques privées, cela révèle le caractère plus ou moins clandestin de ces avortements. En effet, les femmes interrogées ont révélé que le choix des cliniques privées était plus motivé par la discrétion qu’offrent ces structures. Comme l'indiquent les propos d'une interlocutrice, "Dans la clinique, quand tu vas, c'est un peu caché ; personne ne te connait. On ne va pas te poser beaucoup de questions comme à l'hôpital. ». (Mademoiselle M.K, commerçante)

Par ailleurs, le recours à la médecine traditionnelle par 14,71\% des femmes et ce, dans un contexte urbain, cela met en évidence la survivance des pratiques traditionnelles mais surtout la précarité dans laquelle vivent les femmes.

Le choix des tradi-praticiens pourrait également s'expliquer par leur accessibilité financière ${ }^{15}$ en comparaison aux coûts des actes dans les cliniques privées ou dans les structures sanitaires publiques qui se situent entre 25000 francs CFA et 40000 francs CFA selon l'âge de la grossesse.

Les tests effectués ont montré une présomption de liaison entre le lieu d'avortement et la religion : $\left(\chi^{2}=13,888 ; p=0,031\right)$.

\section{Prestataires de soins sollicités pour avorter}

Trois types prestataires de soins ont été identifiés dans le processus d'avortement: les agents de santé du secteur privé, les agents de santé du secteur public et les tradi-praticiens.

$\mathrm{Au}$ niveau des structures sanitaires publiques, les actes sont généralement pratiqués par les jeunes médecins gynécologues en fin de cycle de formation ou nouvellement recrutés à la fonction publique. Ils sont généralement sollicités par des ami(e)s en "difficulté qui veulent se débarrasser d'une grossesse non désirée ». Les actes d'avortement étant délictueux, ils sont pratiqués aux cours des "gardes », ou les jours non ouvrables c'est-à-dire les samedis et dimanches. C'est également l'occasion pour ces jeunes médecins de se faire des sous le temps d'avoir une stabilité financière.

Au niveau des cliniques privées, on retrouve également des jeunes médecins gynécologues en fin de cycle ou nouvellement recrutés, mais aussi des médecins gynécologues confirmés des structures sanitaires publiques. Ces derniers interviennent dans les cliniques privées pour minimiser les risques quand il ne s'agit pas d'un avortement thérapeutique, mais également pour arrondir les fins de mois.

15 En dehors de la consultation dont les coûts varient de 2000 francs CFA à 5000 francs CFA selon les tradi-praticiens, la clientes n'achètent que les médicaments dont les coûts varient également en fonction de l'âge de la grossesse, du type de médicament et du praticien. En général, les coûts varient entre 5000 francs CFA et 10000 francs CFA. 
En dehors des médecins, des infirmiers et sages femmes sont également impliqués dans les pratiques d'avortement. Ces derniers interviennent généralement lorsque l'âge de la grossesse est inférieur ou égale à 12 semaines. Au-delà de cet âge, ils sollicitent l'appui d'un médecin.

Mais dans les cliniques de moindre importance, les infirmiers notamment pratiquent l'avortement quel que soit l'âge de la grossesse.

Au niveau des tradi-praticiens, les prestataires sont généralement des femmes d'un âge avancé, communément appelées «les vieilles » qui sont censées avoir une connaissance et une expérience avérées des plantes médicinales. Elles exercent généralement à domicile pour les consultations et/ou possèdent des étales pour la vente de leurs médicaments.

Les hommes qui sont sollicités sont, plus la plupart, d'origine étrangère. Il s'agit notamment des nigériens et des béninois qui exercent également leurs activités à domicile.

Dans les deux cas, les hommes et les femmes qui exercent le métier de tradi praticiens n'avouent jamais aider les clientes à avorter.

Comme l'indique l'avoue l'une des patientes, "si tu vas les voir pour dire que tu veux avorter, elles ne vont jamais te donner de médicaments. C'est quand vous allez discuter et que tu vas bien expliquer ton problème qu'elles vont t'aider, mais elles vont toujours te dire que si ça ne marche pas, elles ne sont pas responsables » (Dame S. D, vendeuse de friperie).

Ces propos sont corroborés par ceux d'une tradi-praticienne interrogée: "nous on aide les femmes à avoir des enfants, pas pour tuer. Mais quand tu expliques ton problème, on peut t'aider. Ce sont les jeunes filles qui viennent beaucoup nous voir. Les filles sont aussi nos filles, mais quand on voit que si elles gardent la grossesse, ce n'est pas bon pour elles, on essaye de les aider, sinon, moi-même, je ne suis pas d'accord avec avortement. » (Dame K.A, tradi-praticienne dans le quartier de Selmer à Yopougon)

\section{Méthodes utilisées pour avorter}

Les méthodes utilisées pour l'avortement sont fonction des praticiens consultés et du lieu de réalisation de l'avortement. L'analyse des lieux de l'avortement a montré que 58,82\% des avortements ont été réalisés dans les cliniques privées, $17,65 \%$ dans les structures sanitaires publiques, $14,71 \%$ chez les tradi praticiens et 8,82\% à domicile.

Au niveau des structures sanitaires privées et publiques, n’ayant pas eu la possibilité d'observer les plateaux techniques, il est difficile d'apprécier la qualité du matériel. Toutefois, il ressort des entretiens que deux méthodes sont généralement utilisées: l'avortement chirurgicale et l'avortement médicamenteux. 
Pour l'avortement chirurgical, la méthode la plus utilisée est l'avortement par aspiration qui est généralement effectué sous sédation consciente. Cette méthode est généralement appelée «le curetage » par les femmes.

Quant à l'avortement médicamenteux, il est pratiqué quand la période de gestation est inférieure à sept semaines. Deux médicaments sont généralement utilisés pour ce type d'avortement: le méthotrexate et le misoprostol ${ }^{16}$. le méthotrexate est administré par injection et le misoprostol qui sont des comprimés, sont insérer dans le vagin de la patiente cinq à sept jours plus tard.

Au niveau des tradi-praticiens, ce sont généralement les décoctions ou les plantes qui sont prescrites aux femmes.

Pour celles qui ont pratiqué l'avortement à domicile ou l'auto avortement (8,82\%), les méthodes utilisées sont généralement des produits pharmaceutiques conseillés par des amies notamment (le synergon, le crimex, les antipaludéens et antibiotiques surdosés notamment), les produits acides ou sucrés comme la boisson Coca cola mélangée avec d’autres produits pharmaceutiques ou plantes traditionnelles.

Les tests effectués ont montré une présomption de liaison entre la méthode d'avortement utilisée et la religion : $(\chi 2=13,067 ; p=0,042)$.

\section{Discussions}

Notre étude a montré que les avortées sont jeunes et leur âge se situe entre 20 et 34 ans avec un âge moyen de 28 ans. Cette observation a été faite dans d'autres études celles de Konaté et al. (1999) et Guillaume et Desgrées du Loû (2002) qui ont trouvé un âge moyen de 25 ans à Bamako, ce qui est confirmé par l'étude de Coulibaly (op.cit) qui a également trouvé que 62,6\% des avortées de son échantillon avait un âge situé entre 15 et 24 ans. D’auteurs comme Bi Vroh et al (2012) dans une étude sur l'épidémiologie des avortements provoqués en Côte d'Ivoire en 2012 ont montré que les femmes qui ont eu le plus recours à l'avortement avaient moins de 25ans.

Okpani et Okpani (2000) et Guillaume (2003) ont quant à eux montré que l'avortement était beaucoup plus le fait de jeunes femmes en début de vie faconde.

La jeunesse des avortées a également été mise en évidence dans d’autres études antérieures menées en milieu hospitalier en rapport avec les complications d'avortement dans plusieurs capitales africaines (Justesen et

\footnotetext{
${ }^{16}$ Ce médicament augmente les contractions et provoque l'avortement. Les contractions utérines provoquent des douleurs qui ressemblent à celles des règles, parfois plus fortes. Dans 60\% des cas, l'avortement se produit dans les 4 heures suivant la prise du médicament et dans $40 \%$ des cas, l'avortement aura lieu dans les 24 à 72 heures suivant la prise du misoprostol.
} 
al., 1992 ; Ankomah et al., 1997 ; Ezechi et al., 1999 ; Tadesse et al., 2001 ; Goyaux et al., 1999 et 2001). Ces études ont montré que l'âge de la majorité des femmes hospitalisées suite à des complications post-abortum se situait entre 20 et 30 ans.

Les facteurs socioéconomiques ont été des éléments mis en avant par les femmes pour justifier le recours à l'avortement notamment le manque de ressources financières $(69,3 \%)$ et, la stigmatisation et la peur $(41 \%)$. Ces deux raisons sont en effet évoquées dans bon nombre d'études menées sur l'avortement en Afrique. Selon Braam et Hessini (2004), la pauvreté et le manque de moyens financiers constituent l'un des facteurs prépondérant dans la prise de décision d'avorter. Oye-Adeniranet al (2005) ont d'ailleurs indiqué que le risque d'avorter augmente avec le manque d'autonomie financière et matérielle des femmes. D’autres études effectuées au Sénégal (Koly, 1991), au Mozambique (Agadjanian, 1998), en Tanzanie (Mpangile et al., 1999), en Ethiopie (Kebede et al., 2000 et Getahun et Berhane, 2000), en Ouganda (Kasolo, 2000) et au Gabon (Barrère, 2001) et à Madagascar (Gastineau et Rajoanarisoa, 2010) ont également montré que la plupart des femmes qui ont eu recours à l'avortement étaient confrontés à des difficultés économiques. Elles évoquent notamment l'absence ou la faiblesse des leurs revenus pour faire face aux charges d'un enfant.

L'étude de Coulibaly (2009) sur les connaissances, attitudes et pratiques de l'avortement provoqué clandestin en Côte d'Ivoire montre également que $21,3 \%$ des femmes ont dû avorter parce qu'elles ne pouvaient pas assurer la charge d'un enfant supplémentaire.

S’agissant de la peur, elle se résume, dans la plupart des cas, à la peur des parents et à la réprobation de la société. En effet, la peur des parents, la réaction de la famille ou simplement le regard des autres sont déterminants dans la prise de décision de recourir à l'avortement. Barrière (2001) a, dans son étude menée au Gabon, révélé qu’une jeune fille sur cinq âgée de moins de 20 ans a dû avorter parce qu'elle craignait la réaction des parents. Le même constat a été fait dans d'autres études menées en Ouganda (Bazira, 1992), au Bénin (Alihonou, et al.; 1996), au Nigéria (Renne, 1997) et au Togo (URD, 2001) et au Cameroun où la peur d'être rejetée par les parents ou la famille " a été citée comme premier motif d'avortement par 52,8\% des jeunes filles interrogées (Ngwé et al. 2005).

La peur et la stigmatisation vont de pair avec les perceptions liées à l'avortement. En effet les femmes qui avortent sont perçues comme des criminelles, des meurtrières, des prostituées et des pécheresses (JagweWadda et al, 2006 ; N’Bouké, 2011). Une étude menée au Cameroun par Schuster (2005) a d'ailleurs montré que les avortées sont parfois chassées des villages non sans avoir subi les moqueries et les railleries des autres membres de la communauté. 
La proportion des femmes qui a évoqué les motifs scolaires est certes faible dans notre étude (8,82\%), mais il n'en demeure pas moins le désir de poursuivre les études demeure l'un des principaux motifs évoqués par les jeunes filles scolarisés notamment pour avorter (Renne, 1997; Zabin et Kiragu, 1998 et Guillaume, 2004). En Côte d'Ivoire, l'étude de Guillaume (2005) a montré que 55\% des femmes dont 19\% à Abidjan ont indiqué que leur décision d'avorter était liée au désir de poursuivre les études.

La volonté de poursuivre les études a été également mentionnée au Togo par une femme sur trois de moins de 20 ans et $19 \%$ de celles de 20-24 ans (URD, 2001; Djoke, 2004), 22\% des femmes de 15 à 24 ans au Gabonn et 30\% des femmes au Mozambique (Agadjanian, 1998) et par 26\% à 38\% des femmes dans deux régions du Nigeria (Mirembe, 1994, Okonofua et al, 1996)

Notre étude a également montré que les avortements sont pratiqués en majorité en milieu médicalisé par des médecins gynécologues et parfois des infirmiers et sages femmes. Cette observation a été faite dans d'autres études notamment celles de Mpangile et al, en 1993 en Tanzanie, de Ahiadeke en 2001 au Ghana, de Calvès en 2002 au Cameroun et de Singh en 2006 en Ouganda. Ces études ont montré que la plupart des avortements clandestins est le fait de médecins ou infirmiers, mais également du personnel non médical. Toutefois, une frange importante des femmes continuent de solliciter les services des prestataires traditionnels comme en Ouganda, en Tanzanie et au Gabon (Barrière 2001).

Relativement aux méthodes utilisées, notre étude a fait ressortir que les méthodes chirurgicales et médicamenteuses étaient les plus utilisées par les femmes. Cette observation rejoint celles qui ont été faites dans d'autres capitales comme Lomé et Bamako et même à Abidjan où la majorité des études montrent que plus de $60 \%$ des avortements sont pratiqués par des méthodes médicalisées (Konate. et al,1999; Guillaume et Desgrées du Loû, 2002; URD, 2001 ; Guillaume 2005).

D'autres méthodes telles que l'utilisation de médicaments en surdosage ou l'utilisation de plantes et l'introduction d'objets dans l'utérus ont été notées dans d'autres études comme celles de Barrière (2001) au Gabon qui a révélé que le tiers des femmes a pratiqué l'auto-avortement par l'utilisation de plantes médicinales, de médicaments en surdosage ou par l'insertion d'objet dans l'utérus.

Castel (1990) et Likwa (1996) avaient déjà montré qu'en Zambie, les jeunes femmes recouraient à des boissons à base d'essence ou de détergents voire des médicaments en surdosage tels que la chloroquine ou l'aspirine pour avorter quant d'autres utilisaient des méthodes intra utérines dangereuses notamment l'insertion de racines de manioc. 
Ces constats ont également été faits dans les études menées à Abidjan par Goyaux et al (1999) auprès de femmes admises à l'hôpital des suites de complications d'avortement. 31\% des femmes avaient tentés d'introduire des tiges de plantes dans le vagin, $23 \%$ avaient utilisé des ovules végétaux et $20 \%$ des infusions de plantes.

Quant à la prise de décision d'avorter qui est au cœur du processus d'avortement, elle est généralement le fait des femmes elles-mêmes (61,76\%), mais aussi en collaboration avec le partenaire. L'étude de Coulibaly (2009) a montré à cet effet que les partenaires informés de la décision d'avorter ont été dans 58,9\% des cas favorables à l'interruption de la grossesse.

\section{Conclusion}

En Côte d'Ivoire, comme partout ailleurs dans les pays en voie de développement, l'avortement est pratiqué dans un cadre restrictif, ce qui le rend à la fois complexe et controversé. Même si la littérature montre qu'il est beaucoup plus le fait des jeunes femmes, il concerne tous les âges et fait intervenir des dimensions aussi bien politiques, économiques, sociales, religieuses, éthiques que sanitaires.

La majeure partie des études sur l'avortement a été menée en milieu hospitalier et la nôtre est une contribution à la compréhension du phénomène en milieu urbain notamment défavorisé. Des résultats, il ressort que l'avortement se présente comme une pratique complémentaire à la contraception et, le fait que les décisions de recourir à l'avortement soient généralement prises par les femmes elles mêmes, cela dénote d'un besoin latent d'autonomie en matière de reproduction et pourrait constituer un élément déterminant de la compréhension de la pratique à Abidjan et en Côte d'Ivoire.

En effet, l'analyse parallèle des prises de décision et des motivations des femmes a montré que les femmes, même étant soumises aux pressions socioéconomiques (manque de moyens et la peur notamment), elles manifestent le désir d'une certaine autonomie reproductive.

Cette autonomisation des femmes va de paire également avec une certaine négociation reproductive dans la mesure où les décisions d'avorter sont partagées dans la plupart des cas avec les partenaires. De même, le fait que l'avortement devienne un acte concerté, cela peut être également considérer comme le début d'une « déstigmatisation » du phénomène.

Le désir d'autonomie des femmes associé à la négociation entre partenaires et la restriction du cadre législatif et légal ne pourrait-il pas, en définitive, favoriser le recours de plus en plus croissant des femmes à l'avortement? Cette question ouvre ainsi deux champs possibles de recherche qui constituent sans nulle doutent les défis majeurs en matière 
d’avortement provoqué à Abidjan, en Côte d’Ivoire et en Afrique : celui du droit reproductif qui prend en compte de la question de la législation sur l'avortement et celui de l'accès aux avortements sécurisés ou aux soins après avortement qui ont un impact sur la mortalité maternelle.

\section{References:}

1. Agadjanian V., 1998. "Quasi-legal" abortion services in a subSaharan setting: users' profile and motivations." International Family Planning Perspectives 24(3): 111-6.

2. Ahiadeke C., 2001, «Incidence of induced abortion in southern Ghana», International Family Planning Perspectives 27(2): 96-101 \& 108.

3. Alihonou, E., S. Goufodji, et al., 1996. "Morbidity and mortality related to induced abortions (a study conducted in hospitals of Cotonou, Benin in 1993)." African Journal of Fertility, Sexuality and Reproductive Health 1(1): 58-65.

4. Ankomah, A., C. Aloo-Obunga, et al., 1997. "Unsafe abortion: method used and characteristics of patients attending hospitals in Nairobi, Lima and Manila." Health Care for Women International 18: 43-53.

5. Barrère, M., 2001. Avortement. Enquête Démographique et de santé Gabon 2000. F. DGSEE, ORC Macro. Calverton, Maryland, USA : 86-98.

6. Bazira, E. R., 1992. "Induced abortion at Mulago Hospital Kampala, 1983 - 1987: a case for contraception and abortion laws' reform." Tropical Health 11(1): 13-6.

7. Bi Vroh J B et al. 2012 « Épidémiologie des avortements provoqués en Côte d'Ivoire», Santé Publique 2012/HS (Vol. 24), p. 67-76.

8. Braam T. et Hessini L., 2004, «The power dynamics perpetuating unsafe abortion in Africa: A feminist perspective», African Journal of Reproductive Health 8(1): 43-51.

9. Calves, A. E., 2002. "Abortion risk and decision-making among young people in urban Cameroon." Studies in Family Planning 33(3): 249-260.

10. Castle, M. A., R. Likwa, et al. (1990). "Observations on abortion in Zambia." Studies in Family Planning 21(4): 231-5.

11. Coulibaly L. 2009. Connaissances, attitudes et pratiques de l'avortement clandestins en Côte d'Ivoire, communication lors du $\mathrm{XXVI}^{\text {ème }}$ Congrès International de la Population, Marrakech - Maroc, 27 Septembre - 02 Octobre 2009 
12. Desgrées du Loû A., Msellati P., Viho I. et Welffens-Ekra C., 1999 « Le recours à l'avortement dans la ville d'Abidjan : une cause de la baisse de fécondité ? » Population, 54 (3) 1999, 427-446

13. Djoke A, 2004. Recours à l'avortement au Togo: Tendances et Facteurs Explicatifs, Mémoire de DESSD, IFORD, 128 p.

14. Ezechi, O. C., Fasubaa O. B. et al., 1999. "Contraceptive promotion and utilization: solution to problem of illegally induced abortion in countries with restrictive abortion law." Nig. Qt.Hosp. Med. 9(2 april-june 1999): 167-168.

15. Gastineau, B. et Rajaonarisoa, S. Santé de la reproduction et avortement à Antananarivo (Madagascar) Résultats d'une recherché originale. African Journal of Reproductive Health Sept. 2010; 14(3): 223

16. Getahun, H. and Y. Berhane (2000). "Abortion among rural women in north Ethiopia." International Journal of Gynecology and Obstetrics 71(3): 265-6.

17. Guillaume A., 2000. "L'avortement en Afrique : mode de contrôle des naissances et problème de santé publique”, La chronique $d u$ CEPED, vol. n³7, p.3

18. Goyaux, N., F. Yace-Soumah, et al., 1999. "Abortion complications in Abidjan (Ivory Coast)." Contraception 60(2): 107-109.

19. Goyaux, N., E. Alihonou, F. Diadhiou, R. Leke, et P. Thonneau. 2001. "Complications of induced abortion and miscarriage in three african countries: a hospital-base study among WHO collaborating centers."Acta obstetrica et Gynecologica Scandinavica 80: 568-573.

20. Guillaume, A. 2003, Le rôle de l'avortement dans la transition de la fécondité à Abidjan au cours des années 1990, Population (Fr), INED, 2003, 58 (6), 741-771

21. Guillaume A., 2004, L'avortement en Afrique: Une revue de la littérature des années 1990 à nos jours, CEPED, Groupement d'intérêt scientifique, INED-PARIS 2004, CD ROM.

22. Guillaume, A. 2005: L’avortement provoqué en Afrique: un problème mal connu, lourd de conséquence, Laboratoire, Population, Environnement et Développement, Série Santé de la Reproduction, Fécondité et Développement, Document de recherche $n^{\circ} 7, \operatorname{IRD}$, Université de Provence, 24 p.

23. Guillaume A et Desgrées du Loû A, 2002. Limitation des naissances parmi les femmes d'Abidjan, Côte d'Ivoire: contraception, avortement ou les deux? in Perspectives Internationales sur le Planning Familial, numéro spécial de 2002, pp. 4-11

24. Guttmacher Institute, 2009. Les faits sur l'avortement et les grossesses non planifiées en Afrique 
(http://www.guttmacher.org/pubs/IB_AWW-Africa-FR.pdf), site consulté le 02 octobre 2015

25. Hord C.E. et al., 2006, «Unsafe abortion in Africa: an overview and recommendations for action», Pp. 115-149 in Preventing Unsafe Abortion and its Consequences: Priorities for Research and Action, Ina K. WarrineretIqbal H. Shah (Eds). NewYork, Guttmacher Institute 243p.

26. Institut National de la Statistique (INS) et Ministère de la Lutte contre le Sida [Côte d'Ivoire] et ORC Macro. 2006. Enquête sur les Indicateurs du Sida, Côte d'Ivoire 2005. Calverton, Maryland, U.S.A. : INS et ORC Macro, 263 p.

27. Institut National de la Statistique (INS) et ICF International. 2012. Enquête Démographique et de Santé et à Indicateurs Multiples de Côte d'Ivoire 2011-2012. Calverton, Maryland, USA : INS et ICF International.

28. Jagwe-Wadda G. et al., 2006, «Abortion Morbidity in Uganda: Evidence from Two Communities» Occasional Report $N^{\circ} 26$, New York, Guttmacher Institute, 58p.

29. Justesen, A., S. H. Kapiga, et al. 1992. "Abortion in Hospital setting: hidden realities in Dar es Salaam Tanzania." Studies in Family Planning 23(3): 325-329.

30. Kasolo, J., 2000. "Abortion in Uganda." Initiatives in Reproductive Health Policy 3(2): 9-10.

31. Kebede, S., C. Jira, et al. 2000. "A survey of illegal abortion in Jimma Hospital, south western Ethiopia." Ethiop Med J 38(1): 35-42.

32. Koly, F. 1991. L'avortement provoqué clandestin: aspects actuels et perspectives à la clinique gynécologique et obstétricale du CHU de Dakar. Dakar, Faculté de Médecine: 117.

33. Konate, M. K., F. Sissoko, et al. 1999. Les conséquences sociales de l'avortement provoqué à Bamako. Bamako, CILSS, INSAH, CERPOD: 91.

34. Likwa, R. N. and M. Whittaker (1996). "The characteristics of women presenting for abortion and complications of illegal abortions at the University Teaching Hospital, Lusaka, Zambia: an explorative study."African Journal of Fertility, Sexuality and Reproductive Health 1(1): 42-9.

35. Mirembe, F. M. (1994). "A situational analysis of induced abortions in Uganda."

36. Mpangile, G. S., M. T. Leshabari, et al. 1993. "Factors associated with induced abortion in public hospitals in Dar es Salaam, Tanzania." Reproductive Health Matters (2): 21-31. 
37. Mpangile, G., M. Leshabari, et al., 1999. Induced Abortion in Dar es Salaam, Tanzania: The Plight of Adolescents.In Abortion in the developing world.A I Mundigo and C. Indriso. New Delhi, World Health Organization: 387-405.

38. N’Bouké A. Recours à l'avortement provoqué à Lomé (Togo) : évolution, facteurs associés et perceptions. Thèse de doctorat, 2011; Université de Montréal, 227p.

39. Ngwe et al. 2005. Connaissances, Attitudes et Pratiques relatives à l'avortement à Yaoundé et Douala, CCRA, ALVF, 86 p.

40. Okonofua, F. E., C. Odimegwu, et al. (1996). "Women's experiences of unwanted pregnancy and induced abortion in Nigeria. Summary report."

41. OMS, Unsafe Abortion: Global and Regional Estimates of the Incidence of Unsafe Abortion and Associated Mortality in 2003, fifth ed., Geneva: WHO, 2007.

42. Okpani, A. and J. Okpani, 2000. "Sexual activity and contraceptive use among female adolescents-a report from Port Harcourt, Nigeria."African Journal of Reproductive Health 4(1) : 40-48.

43. Oye-Adeniran B.A. et al. 2005, «Induced abortion in Nigeria: Findings from focus group discussion», African Journal of Reproductive Health 9(1): 133-141.

44. Plummer M.L. et al., 2008, «Aborting and suspending pregnancy in rural Tanzania: An ethnography of young people's beliefs and practices», Studies in Family Planning 39(4): 281-292.

45. Renne, E. P. (1997). "Changing patterns of child-spacing and abortion in a northern Nigerian town."

46. Schuster S., 2005, "Abortion in the moral world of the Cameroon grassfields»,Reproductive Health Matters 13(26): 130-138.

47. Silberscmidt M et Rasch V, 2001 “Adolescent girls, illegal abortions and "sugar-daddies"' in Dar es Salaam: vulnerable victims and active social agents”, Social Science \& Medicine 52 (2001) 1815-1826

48. Singh S et al., Unintended Pregnancy and Induced Abortion in Uganda: Causes and Consequences, New York: Guttmacher Institute, 2006.

49. Tadesse, E., G. Yoseph A., et al., 2001. "Illegal abortion in Addis Ababa, Ethiopia." East African medical journal 78(1),: 25-29.

50. URD (Unité de Recherche Démographique), 2001. Avortement au Togo. Famille, migration et urbanisation au Togo. Résultats de l'enquête quantitative. Lomé, Togo, URD, DGSCN: 13.

51. Zabin L.S., Kiragu 1998. The health consequences of adolescent sexual and fertility behaviour in Sub-Saharan Africa, Studies in Family Planning, 29(2), p. 210-232. 